\title{
VARIABLES QUE AFECTAN LA TASA DE INCUMPLIMIENTO DE CREDITOS DE LOS CHILENOS
}

VARIABLES WHICH AFFECT DEFAULT RATE OF CHILEAN LOANS

\section{FRANCISCO ORMAZABAL C.*}

Superintendencia de Bancos e Instituciones Financieras de Chile

\begin{abstract}
This paper contributes relevant empirical evidence which indicates the existence of difference in default rate of loans between male and female. It concludes that independent of types of loans, the default rate of female is less than male. Moreover this paper analyzes the effect of other variables as Married Status and Size/Income over default rate.
\end{abstract}

Keywords: Default, Loans, Gender, Married Status, Income, Consumption Loan, Mortgage loans, Commercial loans.

JEL Classification: E42, E43, E44, E51, G21.

\section{Resumen}

El presente trabajo aporta evidencia empírica relevante que indica la existencia de diferencias en las tasas de incumplimiento crediticio de hombres y mujeres. Se concluye que, para todos los segmentos crediticios, la tasa de incumplimiento del segmento de mujeres es inferior a la exhibida por el segmento de clientes hombres. Al mismo tiempo, se analiza el efecto sobre la tasa de incumplimiento de variables como estado civil y tamaño/ingreso.

Palabras clave: Default, Créditos, Género, Estado Civil, Ingreso, Crédito de Consumo, Crédito para vivienda, Crédito comercial.

Clasificación JEL: E42, E43, E44, E51, G21.

* Las opiniones y errores son de exclusiva responsabilidad del autor.

E-mail: formazabal@sbif.cl 


\section{INTRODUCCION}

La "probabilidad de incumplimiento" (tasa de default) es una de las variables críticas consideradas por las instancias de evaluación crediticia de las instituciones financieras (comités de crédito y de riesgo) al momento de definir la aprobación y las condiciones de crédito aplicables (monto, tasa, plazo y garantías) a una solicitud de financiamiento ${ }^{1}$.

Una serie de investigaciones previas sugieren que el comportamiento de pago de los individuos (y la tasa de default asociada) depende tanto de variables de tipo financiero (en general vinculadas al ingreso) como de variables de carácter personal (edad, estado civil y género, entre otras).

La identificación de los factores de riesgo y la cuantificación de la tasa de default de las personas, son aspectos que inciden en la gestión crediticia de las instituciones financieras, en la estabilidad de las mismas y en su contribución al desarrollo financiero de la economía. En efecto:

- Un adecuado tratamiento de dichas variables contribuye a la mantención de un acceso estable al financiamiento de bienes de consumo durable, cubrir desfases de caja (imprevistos) o simplemente a adelantar consumo futuro.

- Una inadecuada evaluación de lo anterior puede generar efectos negativos relevantes, como la asignación de tasas de interés mayores o la ocurrencia de tasas de incumplimiento inesperadamente altas.

En dicho contexto y en relación con el presente estudio se destaca que:

- Se busca evaluar para el caso chileno la existencia de evidencia empírica sobre la incidencia de variables financieras y personales en la tasa de default de los deudores. Se subraya que no se persigue ahondar en las razones por las cuales diferentes segmentos de deudores diferenciados por atributos como tamaño, género, edad, estado civil e ingreso pueden exhibir distintas tasas de default, sino simplemente corroborar y cuantificar la importancia de tales variables.

- Se evalúa información asociada al universo de personas naturales que suscribieron créditos con instituciones bancarias (durante junio de 2009) y se efectúa un seguimiento de los mismos durante los doce meses siguientes.

- Respecto de trabajos previos reportados por la literatura especializada, el presente estudio no solo presenta un mayor alcance al incluir al universo de deudores, también presenta una mayor precisión al incluir resultados diferenciados según tipo de cartera (consumo, vivienda y comercial²).

1 En este contexto se entenderá que la probabilidad de incumplimiento corresponde a la probabilidad de ocurrencia del evento de incumplimiento. Operativamente, se considera que un deudor es incapaz de honrar sus obligaciones (en la forma pactada) cuando presenta un atraso de 90 días o más.

2 A nivel local existe una participación no menor de las personas naturales en la cartera comercial bancaria. 


\section{UNA BREVE RESEÑA DE LA LITERATURA ESPECIALIZADA}

La ciencia económica (y las finanzas como parte de ella) ha empezado crecientemente a introducir la variable género dentro de las categorías de análisis.

En una crítica a la escasa atención que la economía le daba al género, algunos autores empezaron a argumentar ${ }^{3}$. "El homo economicus no es de sexo indefinido: el personaje que realiza las elecciones racionales entre ocio y renta, sin duda es un varón, que no tiene que cuidar personalmente de sus hijos o de su hogar...". Con ello, se enfatiza la posibilidad de que el comportamiento económico de hombres y mujeres sea diferente como consecuencia de los distintos roles que socialmente se les asigna.

En este ámbito, una de las temáticas que han concitado mayor atención en la literatura especializada es el estudio de las diferencias en el reembolso de las obligaciones crediticias entre hombres y mujeres, particularmente en el contexto microfinanciero.

D’Espallier-Guérin-Mersland (2009) presentan una extensa revisión de la literatura empírica sobre género y reembolso crediticio, de la cual se concluye que la evidencia sobre la materia no es del todo concluyente. En palabras de dichos autores, "La relación entre el género y el reembolso se ha analizado en múltiples estudios. Sin embargo, la evidencia es mixta ${ }^{4}$, y por lo general anecdótica o muy limitada en términos de su alcance geográfico y/o institucional". No obstante lo anterior, el mismo trabajo presenta nueva evidencia empírica de carácter más amplio aunque circunscrito al ámbito del microfinanciamiento, que apoya la hipótesis de una tasa de incumplimiento menor en el caso de las mujeres.

La literatura también aporta algunos argumentos que intentan explicar el origen de las diferencias observadas entre género en relación con las tasas de incumplimiento y reembolso de créditos ${ }^{5}$ :

(i) Las mujeres son más conservadoras o prudentes en sus estrategias de inversión, y por lo tanto tienden a presentar mejores tasas de reembolso crediticio;

(ii) Las mujeres tienen menos acceso a fuentes alternativas de crédito, por lo que para asegurar un acceso continuo al financiamiento, tienden a exhibir mejores tasas de reembolso; y

(iii) Las gestiones de seguimiento y cobranza tienen un mayor impacto en las mujeres debido a que este grupo tiende a mantener un mayor contacto con la institución de financiamiento, tiende a tener menor movilidad (laboral y geográfica) y exhibir mejores respuestas frente a acciones de cobranza activa.

\footnotetext{
Ver Castaño (1999).

4 Se refiere a que existe evidencia tanto apoyando la hipótesis de una menor tasa de incumplimiento por parte de las mujeres, como de la no existencia de diferencias en el comportamiento de pago entre ambos géneros.

5 Todd (1996); Rahman (2001); Goetz y Gupta (1996); y Aghion y Morduch (2000). Todos citados por D’Espallier-Guérin-Mersland (2009).
} 


\section{METODOLOGIA}

\subsection{Consideraciones generales}

El análisis contempla a personas naturales individualmente consideradas (con y sin giro), que durante junio de 2009 suscribieron obligaciones efectivas al día (sin presentar impagos en las obligaciones vigentes previamente suscritas).

Para estimar la probabilidad de incumplimiento ${ }^{6}$ se utiliza la definición de default propuesta por el Comité de Supervisión Bancaria de Basilea, es decir, operaciones con una mora de más de 90 días.

Respecto del horizonte de tiempo a utilizar se siguen las recomendaciones del Comité de Supervisión Bancaria de Basilea, el que sugiere para efectos de tales estimaciones la consideración de un horizonte temporal de 12 meses $^{7}$.

\subsection{Antecedentes del modelo}

Para la estimación de las probabilidades de incumplimiento se utiliza un modelo probabilístico no lineal de elección binaria, donde la variable dependiente puede tomar valores 0 o 1 .

La estimación e interpretación de los modelos probabilísticos lineales plantean una serie de problemas que han llevado a la búsqueda de modelos alternativos que permitan estimaciones más fiables de las variables dicotómicas. Al respecto se indica que:

- Para evitar que la variable endógena estimada pueda situarse fuera del rango $(0,1)$ se ha optado por utilizar un modelo de probabilidad no lineal.

- Específicamente se emplea una función de distribución normal tipificada, la cual da lugar a un modelo Probit donde la variable endógena se relaciona con las variables explicativas a través de esta función de distribución. Esta aproximación a la probabilidad de incumplimiento por medio de un modelo Probit es habitual en la literatura ${ }^{8}$, lo novedoso del ejercicio presentado dice relación con la especificación de las variables independientes utilizadas y con la numerosa cantidad de observaciones empleadas.

La función de distribución normal tipificada se especifica en los siguientes términos:

$$
Y_{i}=\int_{-\infty}^{\alpha+\beta X_{i}} \frac{1}{(2 \pi)^{1 / 2}} e^{\frac{s^{2}}{2}} d s+\varepsilon_{i}
$$

6 Un supuesto clave detrás del análisis es que los individuos que caen en incumplimiento no salen de dicha situación.

7 Se asume que las instituciones financieras reevalúan a sus clientes cada un año.

8 Alfaro, Gallardo y Stein (2010). 
donde la variable $s$ es una variable "aleatoria de integración" con media cero y varianza uno.

En el modelo Probit los resultados se pueden interpretar en términos probabilísticos, de modo que el signo de los parámetros asociados indica la dirección en que se mueve la probabilidad cuando aumenta la variable explicativa correspondiente. No obstante lo anterior, la cuantía del parámetro no coincide con la magnitud de la variación en la probabilidad (como sí ocurre en modelos lineales).

Por otra parte, en este tipo de modelos, al asumir una relación no lineal entre las variables explicativas y en la probabilidad de incumplimiento, se observa que frente a aumentos unitarios en una variable explicativa, se generan variaciones en las probabilidades que no siempre son iguales, ya que dependen del nivel original de la misma.

Dado lo anterior, se ha optado por estimar los efectos marginales asociados a cada una de las variables explicativas, con lo cual la cuantía en la que se ve afectada la probabilidad de incumplimiento se obtiene de forma directa.

\subsection{Especificación del modelo para personas naturales sin giro}

Pese a que en la literatura lo más utilizado para la estimación de tasas de incumplimiento son los modelos Probit, también se realizaron estimaciones con funciones Logit, arrojando resultados no cualitativamente diferentes, los cuales no son reportados en este documento.

Por tanto considerando las definiciones anteriores y usando el Criterio de información ${ }^{9}$ de Akaike (AIC) y Schwarz (BIC) para una selección de variables del tipo forward, los resultados de los criterios de información están descritos en el Cuadro 1.

CUADRO 1.A

CRITERIOS DE INFORMACION PARA SELECCION DE VARIABLES (CONSUMO)

\begin{tabular}{|c|c|c|c|c|c|}
\cline { 2 - 6 } \multicolumn{1}{c|}{} & Intercepto & +Género & +Estado Civil & +edad & $\begin{array}{c}\text { +Decil de } \\
\text { Ingreso }\end{array}$ \\
\hline AIC & 348.1060 & 335.9603 & 334.5954 & 331.8691 & 320.9462 \\
BIC & 341.3337 & 290.5442 & 278.7954 & 267.7380 & 261.8808 \\
\hline
\end{tabular}

9 La selección de modelos mediante criterios de información es una técnica estadística usada para evaluar la calidad relativa de un modelo, controlando situaciones en que la "calidad del ajuste" pudiera estar siendo distorsionada por la incorporación de variables explicativas adicionales. En las dos técnicas de uso habitual para estos casos (Akaike y Schwarz), la medida empleada es una función dependiente de la función de máxima verosimilitud y penaliza por el número de regresores estimados. Formalmente, los criterios se definen como: (a) $\mathrm{AIC}=-2(l / T)+2(k / T)$ y (b) $\mathrm{BIC}=-2(l / T)+k \log (T) / T$, donde $l$ es el valor del logaritmo de la función de máxima verosimilitud, $k$ es el número de regresores del modelo y $T$ es el número de observaciones incluidas en la estimación. En ambos casos, el criterio indica seleccionar el modelo con los valores más bajos para cada criterio. 


\section{CUADRO 1.B}

\section{CRITERIOS DE INFORMACION PARA SELECCION DE VARIABLES (VIVIENDA)}

\begin{tabular}{|c|c|c|c|c|c|}
\cline { 2 - 6 } \multicolumn{1}{c|}{} & Intercepto & +Género & +Estado Civil & +edad & $\begin{array}{c}\text { +Decil de } \\
\text { Ingreso }\end{array}$ \\
\hline AIC & 300.2824 & 277.1828 & 268.4148 & 238.6985 & 256.2011 \\
BIC & 268.9556 & 263.8895 & 253.4386 & 249.6729 & 237.4935 \\
\hline
\end{tabular}

Por tanto, para la cartera de créditos de consumo como para la de vivienda se estima una función con deciles de ingreso estimados en forma endógena, siendo la ecuación a estimar ${ }^{10}$ :

$$
\begin{aligned}
& \text { Default }_{i, j}=\beta_{0}+\beta_{1} \text { Genero }_{i, j}+\sum_{z=2}^{5} \beta_{z} \text { Estado_civil }_{z, i, j}+ \\
& \sum_{w=6}^{15} \beta_{w} \text { Decil__ngreso }_{w, i, j}+\beta_{16} \text { Edad }_{i, j}+\varepsilon_{i, j}
\end{aligned}
$$

donde:

$\mathcal{E}_{i, j}$ es el término de error, el cual se distribuye normal

$$
\text { Default }_{i, j}=\left[\begin{array}{l}
1 \rightarrow \text { Morosidad }>90 \_ \text {días } \\
0 \rightarrow \text { Morosidad }<90 \_ \text {días }
\end{array}\right]
$$

$$
\text { Género }_{i, j}=\left[\begin{array}{l}
1 \rightarrow \text { Mujer } \\
0 \rightarrow \text { Hombre }
\end{array}\right]
$$

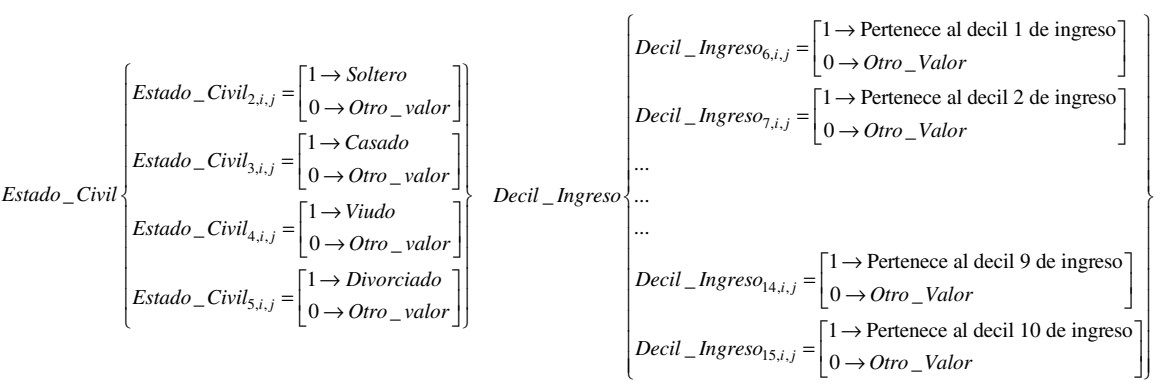

para cada Individuo $i$ que posee un crédito $j$ (Consumo o Hipotecario, según corresponda).

10 La complementación de rentas (por parte de las parejas) es una práctica extendida al momento de acreditar requisitos de ingreso frente a las instituciones financieras (particularmente en los procesos de aprobación de operaciones hipotecarias para la vivienda). No obstante lo anterior, y asumiendo los posibles sesgos asociados, se ha supuesto que la capacidad de pago efectiva de cada agente (hombre o mujer) depende exclusivamente del propio ingreso (supuesto simplificador). 


\subsection{Especificación del modelo para personas naturales con giro (o actividad comercial)}

En el caso de las personas naturales con giro, enmarcadas dentro de la cartera comercial del sistema, se usó el mismo procedimiento de las carteras de consumo y vivienda, y se procedió mediante selección de variables tipo forward y usando los criterios de información de Akaike (AIC) y Schwarz (BIC) (Cuadro 2) al seleccionar un modelo de probabilidad de incumplimiento ajustada por tamaño de empresa deudora ${ }^{11}$.

\section{CUADRO 2}

CRITERIOS DE INFORMACION PARA SELECCION DE VARIABLES (COMERCIAL)

\begin{tabular}{|c|c|c|c|c|c|}
\cline { 2 - 6 } \multicolumn{1}{c|}{} & Intercepto & +Género & +Estado Civil & +edad & $\begin{array}{c}\text { +Tamaño } \\
\text { Empresa }\end{array}$ \\
\hline AIC & 380.4263 & 356.8358 & 343.7869 & 336.9360 & 321.8361 \\
BIC & 283.3149 & 258.5582 & 248.5962 & 246.3334 & 237.2685 \\
\hline
\end{tabular}

La ecuación a estimar sigue la siguiente especificación ${ }^{12}$ :

$$
\begin{aligned}
& \text { Default }_{i, c}=\beta_{0}+\beta_{1} \text { Fenero }_{i, c}+\sum_{z=2}^{5} \beta_{z} \text { Estado_civil }_{z, i, c}+ \\
& \sum_{w=6}^{10} \beta_{w} \text { Tamaño }_{w, i, c}+\beta_{11} \text { Edad }_{i, c}+\varepsilon_{i, c}
\end{aligned}
$$

donde:

$$
\text { Tamaño }\left\{\begin{aligned}
\text { Tamaño }_{6, i, j} & =\left[\begin{array}{l}
1 \rightarrow \text { Microempresas } \\
0 \rightarrow \text { Otro_Valor }
\end{array}\right] \\
\text { Tamaño }_{7, i, j} & =\left[\begin{array}{l}
1 \rightarrow \text { Pequeñas_Empresas } \\
0 \rightarrow \text { Otro_Valor }
\end{array}\right] \\
\text { Tamaño }_{8, i, j} & =\left[\begin{array}{l}
1 \rightarrow \text { Medianas_Empresas } \\
0 \rightarrow \text { Otro_Valor }
\end{array}\right] \\
\text { Tamaño }_{9, i, j} & =\left[\begin{array}{l}
1 \rightarrow \text { Grandes_Empresas } \\
0 \rightarrow \text { Otro_Valor }
\end{array}\right] \\
\text { Tamaño }_{10, i, j} & =\left[\begin{array}{l}
1 \rightarrow \text { Megas_Empresas } \\
0 \rightarrow \text { Otro_Valor }
\end{array}\right]
\end{aligned}\right\}
$$

para cada Individuo “i” que posee un crédito comercial.

11 Se utiliza la parte de la cartera comercial que corresponde a personas naturales con giro o actividad económica, usando una clasificación de ventas propuestas por CORFO, proyectada a los volúmenes de deuda comercial informados a través del Sistema de Deudores SBIF. Las empresas se clasifican en Microdeudor (deuda menor que 500 U.F.), Deudor Pequeño (deuda desde 500 U.F. a 4.000 U.F.), Deudor Mediano (Deuda desde 4.000 U.F. a 18.000 U.F.), Deudor Grande (Deuda desde 18.000 U.F. a 200.000 U.F) y Mega Deudor (Deuda superior a 200.000 U.F.).

12 Limitaciones de las estimaciones: la estimación de las tasas de incumplimientos se limita solo al periodo en análisis, el cual puede estar fuertemente influenciado por la crisis financiera que afectaba en esos días a la economía chilena. Por tanto, las tasas estimadas de incumplimiento pueden estar sobrestimadas si es que se desea ver como una tasa de largo plazo. 


\section{RESULTADOS}

\subsection{Tasas de incumplimiento estimadas: efectos}

Los resultados obtenidos a partir de las regresiones especificadas previamente (ver Anexo 1) permiten identificar tres tipos de efectos: Efecto Género, Efecto Estado Civil y el Efecto Ingreso (o Tamaño). Cada uno de ellos, con un impacto diferente sobre la probabilidad de default de los deudores.

- Efecto Género: Se observa que en todas las carteras crediticias, las mujeres tienden a presentar una menor probabilidad de default que los hombres. En el caso de la cartera de vivienda la probabilidad de default de las mujeres es 1,96\% menor a la de los hombres.

- Efecto Estado Civil: Las personas naturales casadas evidencian mayores tasas de incumplimiento que los solteros, siendo la diferencia más marcada en la cartera comercial.

- Efecto Ingreso/Tamaño: (i) Respecto de los créditos de consumo, se observa que el quinto decil de ingreso es el que presenta la mayor probabilidad de incumplimiento, y solo el pertenecer a los dos últimos deciles de ingreso mejoraría la tasa de incumplimiento de los individuos; (ii) Respecto de la cartera para la vivienda se observa que, a mayores niveles de ingreso menores son las tasas de incumplimiento en dicha cartera; y (iii) En la cartera comercial se aprecia que mientras mayor sea el tamaño de las empresas, mayores son las tasas de cumplimiento y menor es la tasa de default.

De acuerdo con el efecto marginal calculado, todos los resultados son estadísticamente significativos, y las diferencias de valor entre las distintas categorías de una variable, son estadísticamente diferentes de cero ${ }^{13}$.

\subsection{Tasas de incumplimiento efectivas}

La demostración de que las variables testeadas generan comportamientos disímiles en las tasas de incumplimiento, puede ser complementada mediante el cálculo de la "tasa de incumplimiento efectiva" de la población, es decir, las tasas de incumplimiento real de la población de personas naturales analizada (junio de 2009), con la ventaja de eliminar los efectos negativos del término de error implícito en el ejercicio previo.

13 Por medio del test de Wald se rechaza la hipótesis de que los valores son idénticos. 


\section{Cartera de Consumo}

Para esta cartera los principales resultados obtenidos (Tablas 1 y 2) indican que:

- A nivel agregado, las tasas de incumplimiento efectivas de hombres y mujeres son respectivamente $16,51 \%$ y $15,43 \%$. Con ello, las mujeres presentan una tasa de incumplimiento inferior a los hombres en $1,08 \%{ }^{14}$.

- Las personas viudas son las que evidencian menores tasas de incumplimiento efectivas para ambos géneros, siendo $16,03 \%$ en caso de los hombres y $14,58 \%$ en caso de las mujeres.

- Las personas ubicadas en el quinto decil de ingreso (equivalente a un ingreso promedio anual de US\$ 8.500) son las que evidencian mayores tasas de incumplimiento.

\section{TABLA 1}

TASA DE INCUMPLIMIENTO EFECTIVA: MUJERES/CARTERA CONSUMO

\begin{tabular}{|c|c|c|c|c|c|c|}
\hline & & \multicolumn{4}{|c|}{ Estado Civil } & \multirow[b]{2}{*}{ Mujeres } \\
\hline & & Soltero & Casado & Viudo & Divorciado & \\
\hline \multirow{12}{*}{$\begin{array}{l}\text { Deciles } \\
\text { de } \\
\text { ingreso }\end{array}$} & I & $17,36 \%$ & $19,30 \%$ & $20,26 \%$ & $19,16 \%$ & $18,46 \%$ \\
\hline & II & $11,77 \%$ & $11,18 \%$ & $14,86 \%$ & $8,79 \%$ & $11,34 \%$ \\
\hline & III & $14,58 \%$ & $14,62 \%$ & $12,40 \%$ & $15,38 \%$ & $14,61 \%$ \\
\hline & IV & $15,78 \%$ & $14,88 \%$ & $10,63 \%$ & $18,69 \%$ & $15,31 \%$ \\
\hline & V & $21,90 \%$ & $22,34 \%$ & $23,93 \%$ & $24,50 \%$ & $22,17 \%$ \\
\hline & VI & $16,59 \%$ & $18,26 \%$ & $15,84 \%$ & $20,28 \%$ & $17,50 \%$ \\
\hline & VII & $13,24 \%$ & $15,11 \%$ & $15,84 \%$ & $17,81 \%$ & $14,33 \%$ \\
\hline & VIII & $10,96 \%$ & $12,77 \%$ & $14,79 \%$ & $15,45 \%$ & $12,06 \%$ \\
\hline & IX & $8,85 \%$ & $10,77 \%$ & $8,59 \%$ & $14,18 \%$ & $10,08 \%$ \\
\hline & $\mathrm{X}$ & $9,37 \%$ & $8,89 \%$ & $7,14 \%$ & $8,98 \%$ & $9,01 \%$ \\
\hline & Total & $15,22 \%$ & $15,56 \%$ & $14,58 \%$ & $16,74 \%$ & $15,43 \%$ \\
\hline & $\mathrm{N}$ & 377.812 & 465.675 & 26.359 & 8.786 & 879.633 \\
\hline
\end{tabular}

Nota: Con $\mathrm{N}$ igual al número de observaciones.

14 El modelo de regresión (Anexo 1) arroja resultados similares. En efecto, la tasa de default de los hombres se estima en $16,22 \%$ y la de las mujeres en $15,41 \%$. 


\section{TABLA 2}

TASA DE INCUMPLIMIENTO EFECTIVA: HOMBRES/CARTERA CONSUMO

\begin{tabular}{|c|c|c|c|c|c|c|}
\hline & \multicolumn{4}{|c|}{ Estado Civil } & \multirow[b]{2}{*}{ Hombres } \\
\hline & & Soltero & Casado & Viudo & Divorciado & \\
\hline \multirow{12}{*}{$\begin{array}{c}\text { Deciles } \\
\text { de } \\
\text { ingreso }\end{array}$} & I & $18,67 \%$ & $20,49 \%$ & $21,93 \%$ & $24,93 \%$ & $19,84 \%$ \\
\hline & II & $12,44 \%$ & $13,18 \%$ & $11,67 \%$ & $13,74 \%$ & $12,86 \%$ \\
\hline & III & $16,81 \%$ & $15,30 \%$ & $16,67 \%$ & $17,48 \%$ & $15,78 \%$ \\
\hline & IV & $15,67 \%$ & $14,77 \%$ & $16,14 \%$ & $17,34 \%$ & $15,09 \%$ \\
\hline & $\mathrm{V}$ & $23,41 \%$ & $24,63 \%$ & $22,53 \%$ & $26,94 \%$ & $24,12 \%$ \\
\hline & VI & $18,21 \%$ & $22,49 \%$ & $23,84 \%$ & $24,40 \%$ & $20,83 \%$ \\
\hline & VII & $15,63 \%$ & $18,51 \%$ & $21,33 \%$ & $20,32 \%$ & $17,54 \%$ \\
\hline & VIII & $12,15 \%$ & $15,38 \%$ & $16,23 \%$ & $19,05 \%$ & $14,39 \%$ \\
\hline & IX & $10,13 \%$ & $12,18 \%$ & $10,94 \%$ & $17,75 \%$ & $11,64 \%$ \\
\hline & $\mathrm{X}$ & $9,18 \%$ & $8,23 \%$ & $5,16 \%$ & $12,71 \%$ & $8,39 \%$ \\
\hline & Total & $16,43 \%$ & $16,53 \%$ & $16,03 \%$ & $19,89 \%$ & $16,51 \%$ \\
\hline & $\mathrm{N}$ & 521.741 & 643.076 & 36.401 & 12.134 & 1.213 .350 \\
\hline
\end{tabular}

Nota: Con $\mathrm{N}$ igual al número de observaciones.

\section{Cartera para la vivienda}

Para la cartera de créditos asociada al financiamiento de viviendas, los resultados (Tablas 3 y 4 ) indican que:

- Los niveles de las tasas de incumplimiento efectivas son inferiores a los observados en la cartera de consumo, siendo nuevamente la mujer la que presenta las menores tasas de incumplimiento efectivas ${ }^{15}$, esto es un $4,25 \%$ para hombres y un $3,62 \%$ para mujeres.

- Para ambos géneros, el segundo decil de ingreso presenta las mayores tasas de incumplimiento efectivo, llegando a ser incluso superiores a los observados en la cartera de consumo.

- Los solteros son los que presentan menores tasas de incumplimiento efectivas. En efecto, las tasas de incumplimiento efectivas de hombres y mujeres son de $3,77 \%$ y $2,85 \%$, respectivamente.

15 El modelo de regresión (Anexo 1) arroja los siguientes resultados para las tasas de incumplimiento estimadas: $4,45 \%$ para los hombres y $3,78 \%$ para las mujeres. 


\section{TABLA 3}

TASA DE INCUMPLIMIENTO EFECTIVA: MUJERES/CARTERA VIVIENDA

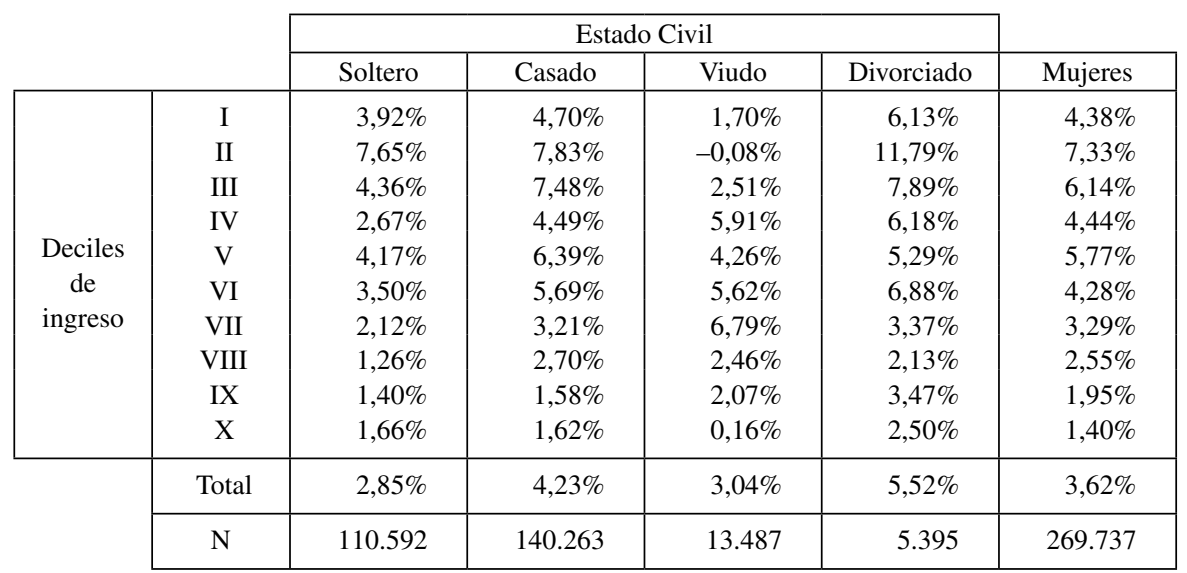

Nota: Con $\mathrm{N}$ igual al número de observaciones.

TABLA 4

TASA DE INCUMPLIMIENTO EFECTIVA: HOMBRES/CARTERA VIVIENDA

\begin{tabular}{|c|c|c|c|c|c|c|}
\hline & & \multicolumn{4}{|c|}{ Estado Civil } & \multirow[b]{2}{*}{ Hombres } \\
\hline & & Soltero & Casado & Viudo & Divorciado & \\
\hline \multirow{12}{*}{$\begin{array}{l}\text { Deciles } \\
\text { de } \\
\text { ingreso }\end{array}$} & I & $4,32 \%$ & $6,85 \%$ & $7,46 \%$ & $11,27 \%$ & $5,90 \%$ \\
\hline & II & $6,97 \%$ & $10,03 \%$ & $19,00 \%$ & $31,63 \%$ & $9,99 \%$ \\
\hline & III & $7,13 \%$ & $9,80 \%$ & $2,03 \%$ & $15,14 \%$ & $8,84 \%$ \\
\hline & IV & $3,02 \%$ & $4,42 \%$ & $3,53 \%$ & $14,81 \%$ & $4,38 \%$ \\
\hline & $\mathrm{V}$ & $6,45 \%$ & $7,76 \%$ & $9,06 \%$ & $5,68 \%$ & $6,93 \%$ \\
\hline & VI & $5,66 \%$ & $7,19 \%$ & $11,20 \%$ & $6,82 \%$ & $7,26 \%$ \\
\hline & VII & $3,35 \%$ & $5,07 \%$ & $4,97 \%$ & $11,91 \%$ & $4,67 \%$ \\
\hline & VIII & $2,01 \%$ & $4,36 \%$ & $3,82 \%$ & $8,37 \%$ & $3,86 \%$ \\
\hline & IX & $0,75 \%$ & $2,00 \%$ & $2,87 \%$ & $3,09 \%$ & $2,46 \%$ \\
\hline & $\mathrm{X}$ & $1,28 \%$ & $1,65 \%$ & $1,33 \%$ & $3,54 \%$ & $1,97 \%$ \\
\hline & Total & $3,77 \%$ & $4,45 \%$ & $4,40 \%$ & $8,60 \%$ & $4,25 \%$ \\
\hline & $\mathrm{N}$ & 146.599 & 185.931 & 17.878 & 7.151 & 357.559 \\
\hline
\end{tabular}

Nota: Con $\mathrm{N}$ igual al número de observaciones. 


\section{Cartera Comercial}

Para el segmento de deudores personas naturales con giro o actividad comercial se observa que (Tablas 5 y 6 ):

- Las empresas de mujeres son las que presentan menores tasas de incumplimiento, siendo esta diferencia poco significativa ${ }^{16}$.

- A mayor tamaño de empresas menores son las tasas de incumplimiento de la empresa, tanto para hombres como mujeres.

- Las mujeres solteras son el segmento que evidencia menores tasas de incumplimiento.

\section{TABLA 5}

TASA DE INCUMPLIMIENTO EFECTIVA: MUJERES/CARTERA COMERCIAL(*)

\begin{tabular}{|c|c|c|c|c|c|c|}
\hline & \multicolumn{4}{|c|}{ Estado Civil } & \multirow[b]{2}{*}{ Mujeres } \\
\hline & & Soltero & Casado & Viudo & Divorciado & \\
\hline $\begin{array}{l}\text { Tamaño } \\
\text { Empresa }\end{array}$ & $\begin{array}{c}\text { Micro } \\
\text { Pequeña } \\
\text { Mediana } \\
\text { Grandes } \\
\text { Megas }\end{array}$ & $\begin{array}{r}20,59 \% \\
14,78 \% \\
15,32 \% \\
8,00 \%\end{array}$ & $\begin{array}{r}34,99 \% \\
16,04 \% \\
14,05 \% \\
10,94 \% \\
0,00 \%\end{array}$ & $\begin{array}{r}31,78 \% \\
12,50 \% \\
19,23 \% \\
0,00 \%\end{array}$ & $\begin{array}{r}38,88 \% \\
19,31 \% \\
8,82 \% \\
16,67 \%\end{array}$ & $\begin{array}{r}27,78 \% \\
15,75 \% \\
14,25 \% \\
10,56 \% \\
0,00 \%\end{array}$ \\
\hline & Total & $19,77 \%$ & $28,53 \%$ & $24,42 \%$ & $33,13 \%$ & $24,73 \%$ \\
\hline & $\mathrm{N}(* *)$ & 69.898 & 82.902 & 5.228 & 4.525 & 162.553 \\
\hline
\end{tabular}

Notas:

(*) Personas naturales con giro o actividad comercial.

(**) N: Número de observaciones.

TABLA 6

TASA DE INCUMPLIMIENTO EFECTIVA: HOMBRES/CARTERA COMERCIAL(*)

\begin{tabular}{|c|c|c|c|c|c|c|}
\hline & \multicolumn{4}{|c|}{ Estado Civil } & \multirow[b]{2}{*}{ Hombres } \\
\hline & & Soltero & Casado & Viudo & Divorciado & \\
\hline $\begin{array}{c}\text { Tamaño } \\
\text { Empresa }\end{array}$ & $\begin{array}{l}\text { Micro } \\
\text { Pequeña } \\
\text { Mediana } \\
\text { Grandes } \\
\text { Megas }\end{array}$ & $\begin{array}{l}25,50 \% \\
17,08 \% \\
16,52 \% \\
11,21 \%\end{array}$ & $\begin{array}{r}35,67 \% \\
16,98 \% \\
15,64 \% \\
14,66 \% \\
0,00 \%\end{array}$ & $\begin{array}{r}33,22 \% \\
16,96 \% \\
21,31 \% \\
0,00 \%\end{array}$ & $\begin{array}{l}42,54 \% \\
23,77 \% \\
26,47 \% \\
20,00 \%\end{array}$ & $\begin{array}{r}32,01 \% \\
17,02 \% \\
15,78 \% \\
14,29 \% \\
0,00 \%\end{array}$ \\
\hline & Total & $23,66 \%$ & $26,32 \%$ & $25,26 \%$ & $35,82 \%$ & $25,64 \%$ \\
\hline & $\mathrm{N}(* *)$ & 104.847 & 124.353 & 7.842 & 6.788 & 243.829 \\
\hline
\end{tabular}

Notas:

(*) Personas naturales con giro o actividad comercial.

(**) N: Número de observaciones.

16 El modelo de regresión (Anexo 1) arroja los siguientes resultados para las tasas de incumplimiento estimadas: $23,75 \%$ para los hombres y $22,00 \%$ para las mujeres. 


\section{CONCLUSIONES}

El modelo de estimación considerado en el estudio muestra la existencia de diferencias materiales en las tasas de incumplimiento estimadas para hombres y mujeres, y entre los diferentes niveles de ingreso o tamaño de empresa.

Según este estudio, son las mujeres las que tienen menores tasas de incumplimiento en todas las carteras crediticias consideradas, lo cual amplía la evidencia de que las mujeres serían mejores pagadoras que los hombres.

El análisis de tasas de incumplimiento efectivas presentado en la parte final del trabajo refuerza las conclusiones derivadas del modelo de regresión. En efecto, no se advierten diferencias significativas en los resultados derivados de ambos tipos de análisis.

Queda para futuras investigaciones realizar un análisis profundo de los fundamentos que explican los resultados encontrados.

\section{BIBLIOGRAFIA}

AGHION, B.A.D. y J. MORDUCH (2000). "Microfinance beyond group lending", Economics of Transition 8, pp. 401-420.

ALFARO, R., N. GALLARDO y R. STEIN (2010). The Determinants of Household Debt Default, Documento de Trabajo Banco Central de Chile 574.

CASTAÑO, C. (1999). "Economía y Género", Política y Sociedad 32, Universidad Complutense de Madrid, pp. 23-42.

D'ESPALLIER-GUERIN-MERSLAND (2009). Women and Repayment in Microfinance, Working Paper 2009-2, Rural Microfinance and Employment.

GOETZ, A. y R.S. GUPTA (1996). "Who takes the credit? Gender, power and control over loan use in rural credit programs in Bangladesh", World Development 24 (1), pp. 45-63.

RAHMAN, A. (2001). Women and Microcredit in Rural Bangladesh: An Anthropological Study of Grameen Bank Lending. Boulder, CO: Westview Press.

TODD, H. (1996). Women at the Center: Grameen Bank Borrowers after One Decade. Dhaka University Press Ltd. 


\section{ANEXO 1}

\section{RESULTADOS MODELO DE ESTIMACION DE LA TASA DE DEFAULT PARA PERSONAS NATURALES}

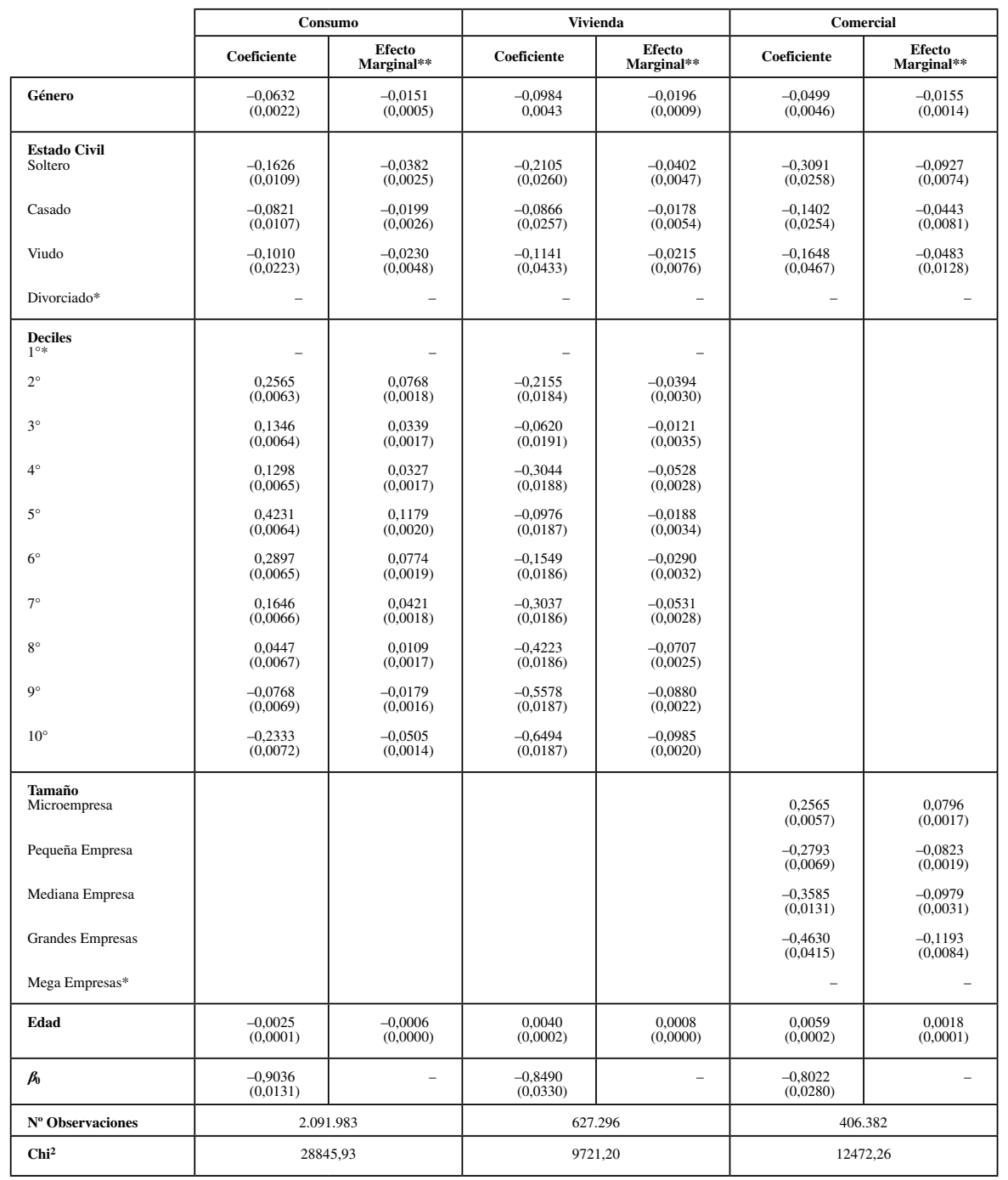

Fuente: Elaboración Propia.

Notas:

* Variables de referencia en el análisis.

** Representa el cambio discreto en la variable Dummy de 0 a 1 (excepto variable Edad). 